\title{
Enteropathogenic and enterohemorrhagic E. coli: ecology, pathogenesis, and evolution
}

\author{
Elizabeth L. Hartland ${ }^{1,2 *}$ and John M. Leong ${ }^{3}$ \\ ${ }^{1}$ Department of Microbiology and Immunology, University of Melbourne, Parkville, VIC, Australia \\ ${ }^{2}$ Murdoch Children's Research Institute, Royal Children's Hospital, Parkville, VIC, Australia \\ ${ }^{3}$ Department of Molecular Biology and Microbiology, Tufts University School of Medicine, Boston, MA, USA \\ *Correspondence: hartland@unimelb.edu.au
}

Edited by:

Yousef A. Kwaik, University of Louisville School of Medicine, USA

Reviewed by:

Yousef A. Kwaik, University of Louisville School of Medicine, USA

The gastrointestinal pathogens enteropathogenic and enterohemorrhagic E. coli (EPEC and EHEC) continue to pose a threat to human health worldwide. While EPEC remains a significant cause of diarrhea in low-income countries, EHEC is more common as a food or water-borne pathogen in industrialized countries. A major difference between EPEC and EHEC is that EPEC has only a human reservoir of infection while EHEC is a zoonotic disease. Strains of EHEC are commensal in many ruminants, particularly cattle, and hence entry into the food chain through fecal contamination of food or water is a risk factor for infection. Another characteristic of EHEC but not EPEC is the production of Shiga toxins, which are associated with the development of severe complications of infection, namely hemorrhagic colitis (HC) and the hemolytic uremic syndrome (HUS). Whereas, HUS can affect patients of any age, EPEC remains a pathogen of infants less than 2 years of age (Robins-Browne and Hartland, 2002).

Despite their differing epidemiology and clinical manifestations, EPEC and EHEC are highly related and share many virulence determinants and features. Both pathogens share a distinctive mechanism of intestinal colonization known as attaching and effacing A/E lesion formation. A/E lesions are characterized by tight attachment of the bacteria to the enterocyte surface, the localized destruction of microvilli and massive ultrastructural changes underneath the adherent bacteria resulting from the accumulation of filamentous actin (Wong et al., 2011). The locus of enterocyte effacement (LEE) is essential for A/E lesion formation, and encodes a type III section system (T3SS) that translocates multiple effector proteins into the infected enterocyte. The LEE is quintessential for the definition of EPEC, as all strains of EPEC carry LEE and induce A/E lesions. The LEE is also used to define some Shiga-toxin producing E. coli (STEC) as EHEC such as O157:H7, although it role in defining EHEC is blurred somewhat by the existence of STEC that lack LEE yet cause HC and HUS and, as in the case of the recent EHEC O104:H4 outbreak, may be highly virulent (Frank et al., 2011). For true A/E pathogens, the LEE is absolutely required for infection and in EHEC O157:H7 its expression appears to be enhanced by passage in the mammalian gut (Brady et al., 2011), suggesting that animal-to-person or person-toperson transmission may be under positive selection during acute infection.
The LEE T3SS system is similar to those found in other pathogens but has an additional filament to the T3SS needle made of polymerized EspA subunits. EspA is essential for translocation to occur and may also provide some adhesive function during bacterial colonization. The most widely accepted model is that the hydrophobic LEE-encoded proteins, EspB and EspD, form a pore in the host cell membrane that provide a conduit for effector translocation through the T3SS. Together EspA, $\mathrm{EspB}$, and EspD constitute the LEE translocon. A recent proposal is that type III secretion is a two-step process whereby surface-localized effector proteins are part of an intermediate effector-translocator complex that precedes effector translocation (Pilar and Coombes, 2011). Future work will be required to reconcile this model with the presence of the EspA filament that structural evidence suggests directly attaches to the T3SS needle (Sekiya et al., 2001).

Among the many translocated effector proteins, Tir plays a critical role in intimate attachment through binding the outer membrane protein intimin. Although intimin and Tir alleles are largely interchangeable despite sequence variation among different $\mathrm{A} / \mathrm{E}$ pathogens, intimin has been associated with additional Tir-independent adhesive functions. This has also been explored as a source of EPEC/EHEC tissue tropism in the past (Fitzhenry et al., 2002). Here a Tir-independent role for intimin in the colonization of streptomycin-treated mice provides more evidence that in some experimental systems, intimin promotes intestinal colonization independently of its role in Tir-binding (Mallick et al., 2012).

While the intimin-Tir interaction has been intensely studied, not all effector proteins contribute to A/E lesion formation and cytoskeletal changes. It has recently emerged that several effector proteins play a role in dampening the inflammatory response. The effector proteins involved are all encoded outside the LEE and have novel enzymatic functions that target NF- $\mathrm{B}$ and MAPK signaling. For example, NleE is a cysteine methyl transferase that methylates the zinc finger domain of $\mathrm{TAB} 2 / 3$, thereby inhibiting TAB2/3 interaction with ubiquitinated TRAF and blocking the phosphorylation of IКB by the IKK complex and hence the degradation of IкB (Newton et al., 2010; Zhang et al., 2011). NleC and NleD are zinc metalloproteases that cleave the p65 subunit of NF- $\kappa$ B and the MAPKs p38 and JNK, respectively (Baruch et al., 2011; Pearson et al., 2011). NleH has both 
anti-apoptotic and anti-inflammatory effects acting in part by binding ribosomal protein S3 (Hemrajani et al., 2010; Gao and Hardwidge, 2011).

Although much research is aimed at understanding the function of the LEE and the translocated effector proteins [recently reviewed in Wong et al. (2011)], the carriage and transfer of virulence genes among different $E$. coli pathogens makes the study of other potential virulence factors critically important. For example, EhaJ is an autotransporter protein that is shared by strains of belonging to both EPEC and EHEC lineages. Autotransporters are highly prevalent in EPEC/EHEC genomes, and many contribute to inter-bacterial interactions and biofilm formation (Wells et al., 2010). EhaJ also has biofilm-producing properties when expressed in laboratory strains of E. coli, and this requires the function of a putative glycosyltransferase encoded by the adjacent gene, egtA. In addition, EhaJ has extracellular matrix binding properties, suggesting that the autotransporter may contribute to host infection. Other factors apart from the T3SS may also contribute to the ability of EHEC O157:H7 to persist and colonize the surface of plants, including pili and flagella (Saldana et al., 2011), as occurred in a large outbreak of EHEC O157:H7 that was spread by contaminated spinach (Wendel et al., 2009).

The reservoir of EHEC in cattle deserves particular attention as many LEE-independent factors, such as autotransporters, may contribute to persistence in this natural host.

\section{REFERENCES}

Bai, J., McAteer, S. P., Paxton, E., Mahajan, A., Gally, D. L., and Tree, J. J. (2011). Screening of an E. coli O157:H7 bacterial artificial chromosome library by comparative genomic hybridization to identify genomic regions contributing to growth in bovine gastrointestinal mucus and epithelial cell colonization. Front. Microbio. 2:168. doi: 10.3389/fmicb.2011. 00168

Baruch, K., Gur-Arie, L., Nadler, C., Koby, S., Yerushalmi, G., Ben-Neriah, Y., et al. (2011). Metalloprotease type III effectors that specifically cleave JNK and NF-kappaB. EMBO J. 30, 221-231.

Brady, M. J., Radhakrishnan, P., Liu, H., Magoun, L., Murphy, K. C., Mukherjee, J., et al. (2011). Enhanced actin pedestal formation by enterohemorrhagic Escherichia coli O157:H7 adapted to the mammalian host. Front. Microbio. 2:226. doi: 10.3389/fmicb.2011.00226

Coombes, B. K., Gilmour, M. W., and Goodman, C. D. (2011). The evolution of virulence in non-o157 shiga toxin-producing Escherichia coli. Front. Microbiol. 2:90. doi: 10.3389/fmicb.2011.00090
Fitzhenry, R. J., Pickard, D. J., Hartland, E. L., Reece, S., Dougan, G., Phillips, A. D., et al. (2002). Intimin type influences the site of human intestinal mucosal colonisation by enterohaemorrhagic Escherichia coli O157:H7. Gut 50, 180-185.

Frank, C., Werber, D., Cramer, J. P., Askar, M., Faber, M., An Der Heiden, M., et al. (2011). Epidemic profile of Shiga-toxin-producing Escherichia coli O104:H4 outbreak in Germany. N. Engl. J. Med. 365, 1771-1780.

Gao, X., and Hardwidge, P. R. (2011). Ribosomal protein s3: a multifunctional target of attaching/effacing bacterial pathogens. Front. Microbio. 2:137. doi: 10.3389/fmicb.2011.00137

Hemrajani, C., Berger, C. N., Robinson, K. S., Marches, O., Mousnier, A., and Frankel, G. (2010). NleH effectors interact with Bax inhibitor-1 to block apoptosis during enteropathogenic Escherichia coli infection. Proc. Natl. Acad. Sci. U.S.A. 107, 3129-3134.

Low, J. C., McKendrick, I. J., McKechnie, C., Fenlon, D., Naylor, S. W., Currie, C., et al. (2005). Rectal carriage of enterohemorrhagic Escherichia coli $\mathrm{O} 157$ in

Indeed recent work reported here on the interactions of EHEC O157:H7 with bovine rectal epithelial cells suggests that the bacteria are internalized though an intimin- and Tirindependent mechanism. The rectum is the main site of EHEC O157:H7 colonization in cattle and acts as an ongoing source of contamination through fecal shedding (Low et al., 2005). To identify factors that contribute to the ability of EHEC to attach to rectal epithelial cells, here Bai et al. screened BAC clones derived from EHEC O157:H7 in a competitionbased assay. They identified fimbriae and the autotransporter EhaA as candidates for EHEC-bovine epithelium interactions (Bai et al., 2011).

Because of its prominence in large outbreaks of disease, much research has focused on EHEC O157:H7 virulence. Nevertheless non-O157 EHEC, of which there are more than 100 serotypes, also have the capacity to cause HUS and we know far less about their genome structure and evolution (Coombes et al., 2011). As this information accumulates, we will have a much greater understanding of the relationship between EHEC/EPEC and other E. coli pathogens that all contribute to a common gene pool from which new pathogens, such as EHEC O104:H4, may emerge. Ongoing surveillance with high throughput genomics and molecular and infection studies are the keys to understanding the ecology and pathogenesis of EPEC and EHEC and are our only weapons against a fast evolving and dynamic group of pathogens.

slaughtered cattle. Appl. Environ. Microbiol. 71, 93-97.

Mallick, E. M., Brady, M. J., Luperchio, S. A., Vanguri, V. K., Magoun, L., Liu, H., et al. (2012). Alleleand tir-independent functions of intimin in diverse animal infection models. Front. Microbio. 3:11. doi: $10.3389 /$ fmicb.2012. 00011

Newton, H. J., Pearson, J. S., Badea, L., Kelly, M., Lucas, M., Holloway, G., et al. (2010). The type III effectors $\mathrm{NleE}$ and $\mathrm{NleB}$ from enteropathogenic E. coli and OspZ from Shigella block nuclear translocation of NF-kappaB p65. PLoS Pathog. 6:e1000898. doi: 10.1371/journal.ppat.1000898

Pearson, J. S., Riedmaier, P., Marches, O., Frankel, G., and Hartland, E. L. (2011). A type III effector protease NleC from enteropathogenic Escherichia coli targets NF-kappaB for degradation. Mol. Microbiol. 80, 219-230.

Pilar, A. V., and Coombes, B. K. (2011) A fresh look at the type III secretion system: two-step model of effector translocation in pathogenic bacteria. Front. Microbio. 2:113. doi: 10.3389/fmicb.2011.00113

Robins-Browne, R. M., and Hartland, E. L. (2002). Escherichia coli as a cause of diarrhea. J. Gastroenterol. Hepatol. 17, 467-475.

Saldana, Z., Sanchez, E., XicohtencatlCortes, J., Puente, J. L., and Giron, J. A. (2011). Surface structures involved in plant stomata and leaf colonization by shiga-toxigenic O157:H7. Front. Microbio. 2:19. doi: 10.3389/fmicb.2011.00119

Sekiya, K., Ohishi, M., Ogino, T., Tamano, K., Sasakawa, C., and Abe, A. (2001). Supermolecular structure of the enteropathogenic Escherichia coli type III secretion system and its direct interaction with the EspA-sheath-like structure. Proc. Natl. Acad. Sci. U.S.A. 98, 11638-11643.

Wells, T. J., Totsika, M., and Schembri, M. A. (2010). Autotransporters of Escherichia coli: a sequence-based characterization. Microbiology 156, 2459-2469.

Wendel, A. M., Johnson, D. H., Sharapov, U., Grant, J., Archer, J. R., Monson, T., et al. (2009). Multistate outbreak of Escherichia coli $\mathrm{O} 157: \mathrm{H7}$ infection associated with consumption of packaged spinach, August-September 2006: the Wisconsin investigation. Clin. Infect. Dis. 48, 1079-1086.

Wong, A. R., Pearson, J. S., Bright, M. D., Munera, D., Robinson, 
K. S., Lee, S. F., et al. (2011). Enteropathogenic and enterohaemorrhagic Escherichia coli: even more subversive elements. Mol. Microbiol. 80, 1420-1438.

Zhang, L., Ding, X., Cui, J., Xu, H., Chen, J., Gong, Y. N., et al. (2011).
Cysteine methylation disrupts ubiquitin-chain sensing in NFkappaB activation. Nature 481, 204-208.

Received: 13 April 2013; accepted: 13 April 2013; published online: 30 April 2013.
Citation: Hartland EL and Leong JM (2013) Enteropathogenic and enterohemorrhagic E. coli: ecology, pathogenesis, and evolution. Front. Cell. Infect. Microbiol. 3:15. doi: 10.3389/fcimb. 2013.00015

Copyright (c) 2013 Hartland and Leong. This is an open-access article distributed under the terms of the Creative Commons Attribution License, which permits use, distribution and reproduction in other forums, provided the original authors and source are credited and subject to any copyright notices concerning any third-party graphics etc. 